Case Report

\title{
Rhinocerebral mucormycosis: a case report
}

\author{
GCS Gunasekera ${ }^{1}$, CGUA Patabendige ${ }^{1}$, PI Jayasekera ${ }^{2}$, RP Dayasena ${ }^{1}$ \\ Sri Lankan Journal of Infectious Diseases 2016 Vol.6 (1):67-70 \\ DOI: http://dx.doi.org/10.4038/sljid.v6i1.8100
}

\begin{abstract}
Rhinocerebral mucormycosis is a life threatening fungal infection occurring in humans, which is caused by the ubiquitous saprophytic fungi of order Mucorales. A timely diagnosis in patients with predisposing factors leading to immunosuppression is of great importance in reducing mortality and morbidity. We describe a patient presenting with typical clinical manifestations of rhinocerebral mucormycosis involving the paranasal sinuses and the orbit.
\end{abstract}

Keywords: Mucormycosis, fungal infection, rhizopus, amphotericin B, surgical debridement

\section{Case report}

A 39 year old male patient presented to a medical ward with a history of fever, left sided earache with discharge and headache for 1 month; swelling, weakness and numbness of left side of the face for 10 days with drooping and difficulty in moving the left eye for 4 days. He was a noncompliant type II diabetes mellitus patient for 9 years and a heavy alcoholic. A diagnosis of left sided otitis media, mastoiditis and sinusitis was made at another institute. A non-contrast CT scan of petrous-temporal bones and paranasal sinuses done due to the facial symptoms revealed acute sinusitis involving the left frontal, maxillary, sphenoid and bilateral ethmoid sinuses and left sided mastoiditis with otitis media.

On examination he was conscious and rational, afebrile and had no signs of meningism but had complete ptosis of the left eye, proptosis and lid oedema, left sided complete ophthalmoplegia and V and VII cranial nerve palsies.

On admission his capillary blood sugar was $293 \mathrm{mg} / \mathrm{dl}$ and WBC was $8600 / \mathrm{mm}^{3}$ (DC- N 53.8\%, L 36.6\%). Oral hypoglycaemics, nasal decongestants, antihistamines and empirical antibiotics were started. An urgent referral was made to the ENT surgery team as mucormycosis was high in the differential diagnoses. A rigid nasal endoscopy (RNE) revealed fungal debris and pus in the left maxillary region and polypoidal lesions lateral to the maxillary turbinate. A biopsy of the latter was sent for histology. The patient was transferred to the ENT ward. Histology report suggested a fungal infection, possibly mucormycosis. Three days later, another RNE and

\footnotetext{
${ }^{1}$ National Hospital of Sri Lanka

${ }^{2}$ Medical Research Institute, Sri Lanka

Address for correspondence: Dr. G.C.S. Gunasekera, Department of Microbiology, National Hospital of Sri Lanka Telephone: +94112691111; E-mail: chathurigcs@yahoo.com
}

Received 23 December 2015 and revised version accepted 2 March 2016 
washout was done via the maxillary osteum. Necrotic material and pus was sent for fungal studies.

Based on the clinical presentation and histopathology, intra-venous amphotericin-B (conventional type) was started and it was justified as the direct microscopy (KOH mount) became positive for broad based 'aseptate' fungal filaments (Figure 1).

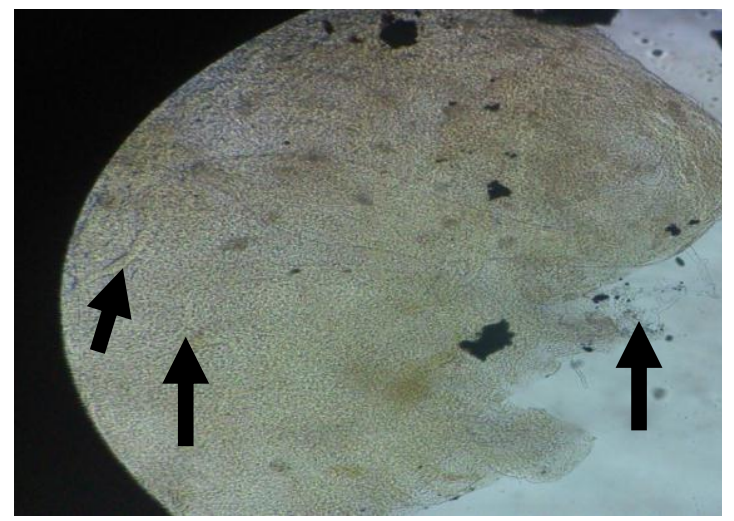

Figure 1: Direct KOH mount showing broad, aseptate fungal filaments

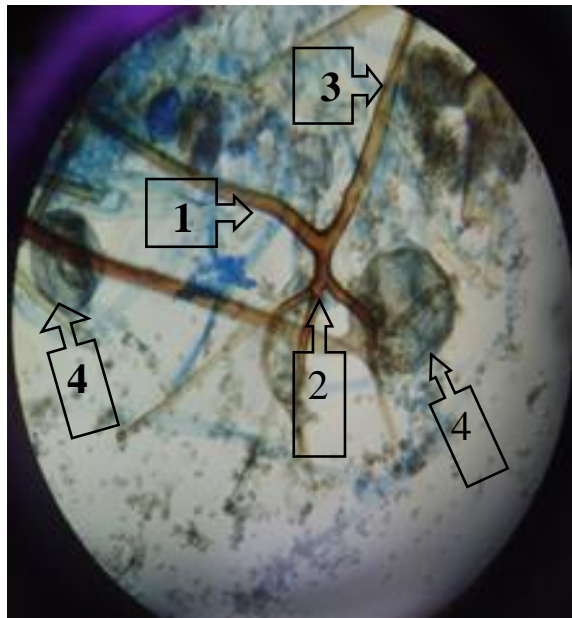

Figure 4: Microscopic features leading to identification of Rhizopus spp. (x40 magnification)

In 2 days, culture in Sabouraud's Dextrose Agar (SDA) yielded a Rhizopus spp. The macroscopic appearance of these colonies is shown in Figures 2 and 3. The microscopic features such as stolons, pigmented nodal rhizoids, sporangiophores and inverted umbrella-like structures formed by collapsed collumellae and apophyses which occur after spore release help in identification of Rhizopus spp. (Figure 4)

1. Stolon

2. Rhizoid

3. Sporangiophore

4. Collapsed columellae

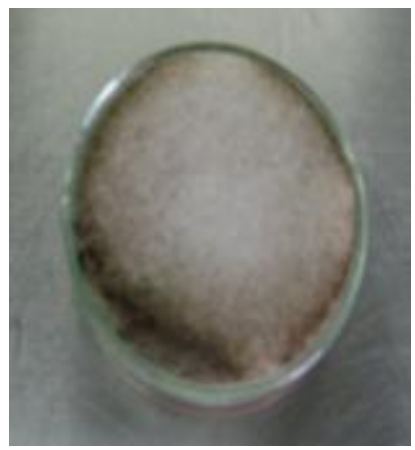

Figure 2: Macroscopic appearance as seen in a SDA plate.

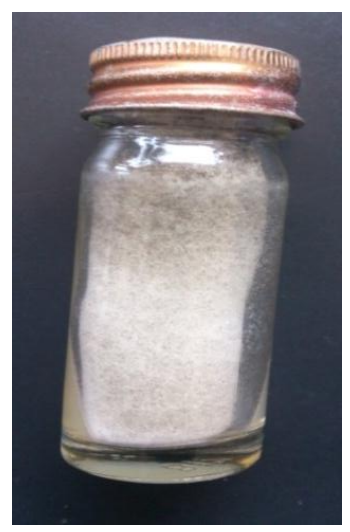

Figure 3: Macroscopic appearance as seen in a SDA slant 
On the $3^{\text {rd }}$ day of amphotericin-B therapy, endoscopic surgery revealed necrotic tissue and fungal matter filled in nasal sinuses. Of these, direct microscopy and histology were positive for fungal filaments but the culture was negative after 7 days of incubation, implying that therapy had started working.

Surgical debridement of the fungal material on four occasions, prompt commencement of systemic anti-fungal therapy with intravenous amphotericin-B and maintaining a good glycaemic control with soluble insulin made the management successful. The adverse effects of the drug were tackled with close monitoring of renal and liver function tests, full blood count and serum

$\mathrm{K}^{2+}$ and $\mathrm{Mg}^{2+}$ levels. Occasionally serum potassium, magnesium and creatinine levels were high but these were rectified with proper replacements and hydration.

After thirty days of systemic amphotericin-B therapy (after a cumulative dose of $1.5 \mathrm{~g}$ of amphotericin B), our patient achieved a profound clinical recovery. The duration of treatment is highly individualized and depends on clinical, radiological and microbiological response.

\section{Discussion:}

Mucormycosis is a group of fungal infections caused by fungi in the subphylum Mucormycotina (order Mucorales). It can cause severe life threatening infections in humans. Many causative agents including Rhizopus spp. are responsible. These ubiquitous fungi in the environment appear as large, ribbon-like hyphae, irregular in diameter with occasional septae ('aseptate'). Spores contained in sporangiospores aerosolize and disperse thus the route of infection is mostly by inhalation. Uncontrolled diabetes mellitus, metabolic acidosis, penetrating trauma, IV drug use, transplantation, malignancy, HIV etc. are known risk factors. In a vast spectrum of clinical manifestations, rhino-cerebral infections are the commonest. Imaging will guide but microbiology and histopathology will confirm the diagnosis and a high level of clinical suspicion is essential. ${ }^{1}$

For successful treatment; timely diagnosis, correction of predisposing factors, repeated surgical debridement and systemic antifungals are required in combination. ${ }^{2}$ Systemic amphotericin-B therapy is complicated with its side effects which need vigilant monitoring. In view of prevention, the risk factors have to be addressed. The highest occurrence of rhinocerebral mucormycosis is in patients with uncontrolled diabetes mellitus. ${ }^{3}$ Therefore, a very good control in diabetes mellitus is of utmost importance to prevent this.

\section{Conclusion}

A high level of clinical suspicion is essential to make a timely diagnosis and comprehensive management includes; correction of predisposing factors, surgical debridement and systemic antifungal treatment. 


\section{References:}

1. Kontoyiannis DP and Lewis RE. Agents of Mucormycosis and Entomophthoramycosis. In: Mandell GL, Bennett JE and Dolin R (eds). Principals and practice of infectious diseases. New York: Churchill Livingstone, 2010; Vol. 1. Ch. 259, $7^{\text {th }}$ edition: pp 3257-3269.

2. Ribes JA., Vanover-Sams CL,. Barker DJ. Zygomycetes in human disease. Clin Microbiol Rev. 2000; 13:236-301. http://dx.doi.org/10.1128/CMR.13.2.236-301.2000

3. Roden MM., Zaoutis TE., Buchanan WL., et al. Epidemiology and outcome of zygomycosis: a review of 929 reported cases. Clin Infect Dis. 2005; 41:634-653.

http://dx.doi.org/10.1086/432579 